\title{
Flow Cytometric Analysis and Isolation of Permeabilized Dopamine Nerve Terminals from Rat Striatum
}

\author{
Marina E. Wolf and Gregory Kapatos \\ Laboratory of Neurochemistry, Center for Cell Biology, Sinai Research Institute, and the Cellular and Clinical Neurobiology \\ Program, Department of Psychiatry, Wayne State University School of Medicine, Detroit, Michigan 48235
}

\begin{abstract}
Fluorescence-activated cell sorting (FACS) was used to identify and isolate permeabilized dopaminergic nerve terminals from rat striatum based on immunofluorescent labeling of tyrosine hydroxylase (TH). Striatal synaptosomes were permeabilized by fixation with modified Zamboni fluid. A highly fluorescent subpopulation of particles was detected by FACS following sequential incubation with a monoclonal antibody to TH (LNC 1) and a fluorescein-conjugated secondary antibody. After correcting for nonsynaptosomal particles present in the synaptosomal fraction, analysis of these data suggested that approximately $12-15 \%$ of striatal synaptosomes were dopaminergic, consistent with previous estimates. Specific labeling by LNC 1 was decreased if synaptosomes were prepared from rats that had received intraventricular injections of 6-hydroxydopamine. The decrease in labeling was highly correlated with the extent of the lesion as determined by measurement of striatal dopamine levels, suggesting that LNC 1-labeled synaptosomes were derived from nigrostriatal dopamine terminals. In order to verify that LNC 1-labeled synaptosomes were enriched for TH, equal numbers of labeled and control synaptosomes were isolated by FACS and analyzed by SDS-PAGE. LNC 1-labeled synaptosomes were shown by Western blot techniques to be enriched 6 -fold for TH compared with control synaptosomes, suggesting that the labeled population consisted almost entirely of dopaminergic synaptosomes.
\end{abstract}

Dopamine (DA) neurons have been the focus of intensive interest because of their involvement in psychiatric and neurological disorders. However, the extent to which the inherent properties of DA neurons can be characterized is limited by the cellular complexity of most currently available experimental preparations. In vitro studies of dopaminergic function, for example, are typically conducted using homogenates, brain slices, or synaptosomes. In each case, a heterogenous population of cells or nerve terminals is analyzed as a group, and results are expressed as an average value per unit of wet weight or protein.

Flow cytometry provides a means of conducting biochemical studies at the single cell level. This approach is advantageous

\footnotetext{
Received Nov. 9, 1987; revised June 2, 1988; accepted June 3, 1988

We thank Tim Geddes and Vickie Kemski for excellent technical assistancz, and Drs. James G. Granneman, Louis A. Chiodo, Michael J. Bannon, and Matthew $P$. Galloway for helpful discussions of the manuscript. We also thank Cynthia Stinson and Kate Early for expert assistance with manuscript preparation. This work was supported in part by U.S. Public Health Service Grant MH-43758. M.W. is the recipient of a National Kesearch Service Award (NS-08413).

Correspondence should be addressed to Dr. Marina E. Wolf, Laboratory of Neurochemistry, Center for Cell Biology, Sinai Research Institute, 6767 West Outer Drive, Detroit, MI 48235

Copyright (C) 1989 Society for Neuroscience $0270-6474 / 89 / 010106-09 \$ 02.00 / 0$
}

in several respects. First, each cell in a population is analyzed individually, so that differences between subpopulations are not obscured. Second, quantitative and multiparameter measurements are made on many thousands of cells, enabling the properties of a population to be defined based on a statistically adequate sample. Finally, subpopulations of interest can be purified for further analysis (for reviews, see Kruth, 1982; Loken and Stall, 1982).

In the accompanying paper, we describe methods for the flow cytometric identification and analysis of striatal synaptosomes (Wolf and Kapatos, 1989). These methods were developed in the course of studies aimed at isolating dopaminergic striatal nerve terminals using fluorescence-activated cell sorting (FACS). The isolation of a subpopulation of intact cells or nerve terminals by FACS requires a fluorescent probe (e.g., a monoclonal antibody) that recognizes a cell surface marker specific to that subpopulation. For example, monoclonal antibodies to cell surface antigens have been used in conjunction with FACS to purify several neuronal populations (Kapatos et al., 1983, 1985; Moskal and Schaffner, 1986). No such probe has yet been developed for DA neurons, although an enrichment of DA neurons from embryonic mouse mesencephalon has been obtained using a monoclonal antibody to neural specific protein 4 (di Porzio et al., 1987). If cells are permeabilized by fixation, however, it becomes possible to analyze and sort based on immunofluorescent labeling of intracellular antigens. This approach has been used successfully in the isolation of neurophysin-containing neurons from rat hypothalamus (Paden et al., 1986).

We now report the isolation of striatal DA nerve terminals using a similar strategy. DA nerve terminals were identified based on the presence of tyrosine hydroxylase (TH), an intracellular antigen specific to DA nerve terminals in this tissue. Selective immunolabeling was accomplished using a monoclonal antibody to TH and a fluorescein-conjugated secondary antibody. DA nerve terminals were then detected and isolated using a fluorescence-activated cell sorter. The availability of pure preparations of fixed DA nerve terminals may enable the identification and quantification of their intracellular and cell surface constituents (e.g., enzymes, structural proteins, receptors, ion channels, and transporters).

\section{Materials and Methods}

Preparation of synaptosomes. Male Sprague-Dawley rats (200-300 gm) obtained from Hilltop Laboratories (Portage, MI) were used in all experiments. Rats were killed by decapitation and the striata dissected over ice. Synaptosomes were prepared according to the method of Gray and Whittaker (1962) as described in the accompanying paper (Wolf and Kapatos, 1989). For incubations and immunolabeling, synaptosomes were used at a concentration of approximately $1 \mathrm{mg}$ protein $/ \mathrm{ml}$ in Kreb's Ringer phosphate buffer (KRP) consisting of $121.9 \mathrm{mM} \mathrm{NaCl}$, 
$0.87 \mathrm{~mm} \mathrm{CaCl}_{2}, 4.89 \mathrm{~mm} \mathrm{KCl}, 1.23 \mathrm{~mm} \mathrm{MgSO}_{4}, 1.23 \mathrm{~mm} \mathrm{KH} \mathrm{PO}_{4}$, $10.3 \mathrm{~mm}$ sodium phosphate buffer (pH 7.4), and $11.87 \mathrm{~mm}$ glucose. Synaptosomes were diluted $1: 100$ in this buffer immediately prior to FACS analysis.

Flow cytometric analysis and sorting. Flow cytometry was performed with a FACS 440 (Becton-Dickinson, Mountain View, CA) using a 5 W argon-ion laser (Spectra Physics) tuned to generate $200 \mathrm{~mW}$ with a $488 \mathrm{~nm}$ emission line. Instrument settings and optics were the same as described in the accompanying paper (Wolf and Kapatos, 1989). Synaptosomal fluorescence was analyzed with logarithmic amplifiers set for $4 \log$ decades.

Fixation of synaptosomes. For most experiments, synaptosomes were fixed with a modified version of Zamboni fluid (Stefanini et al., 1967) as described previously for dissociated cells (Hatfield and Hymer, 1985; Paden et al., 1986). Equal volumes of synaptosomes (in KRP, pII 7.4) and modified Zamboni fluid (4\% wt/vol paraformaldehyde and $15 \%$ $\mathrm{vol} / \mathrm{vol}$ saturated picric acid in $0.1 \mathrm{M}$ phosphate buffer, $\mathrm{pH} 7.4$ ) were combined and incubated for $30 \mathrm{~min}$ at room temperature, yielding final concentrations of $2 \%$ paraformaldehyde and $7.5 \%$ picric acid. For purposes of comparison (see Fig. 6), synaptosomes were also fixed with paraformaldehyde (final concentrations of $0.01,0.1,1$, and $2 \%$ ) in 0.1 $\mathrm{M}$ phosphate buffer ( $\mathrm{pH} \mathrm{7.4)}$ by incubating for $30 \mathrm{~min}$ at room temperature. In all cases, synaptosomes were washed 4 times after fixation by centrifugation $(16,000 \times g$ for $15 \mathrm{~min})$ and resuspension in modified Dulbecco's PBS (modified DPBS; Gibco Laboratories, Grand Island, $\mathrm{NY}$ ) containing $0.53 \mathrm{mM} \mathrm{MgCl}$, and no $\mathrm{CaCl}_{2}$. Fixed synaptosomes may be stored in this solution for several weeks at $4^{\circ} \mathrm{C}$ without significant alteration of their light scattering properties.

Immunofluorescence labeling. TH-containing synaptosomes were identified using a mouse monoclonal antibody (LNC 1) of the $\mathrm{IgG}_{1}$ class directed against TH purified from the $\mathrm{PC} 12$ pheochromocytoma cell line (G. Kapatos and D. Kuhn, in preparation). Synaptosomes were incubated overnight at $4^{\circ} \mathrm{C}$ with $2 \mu \mathrm{g} / \mathrm{ml}$ of $\mathrm{LNC} 1 \mathrm{IgG}$ (equivalent to a 1:2000 dilution of LNC 1 ascites fluid). The secondary antibody, fluorescein isothiocyanate-conjugated (FITC) $F(a b)_{2}$ goat anti-mouse IgG (Jackson ImmunoResearch, Avondale, PA), was used at a dilution of 1:50. Details of the immunolabeling procedure are provided by Wolf and Kapatos (1989). Ascites fluid containing mouse IgG from either of 2 adjuvant-induced plasmacytoma lines, RPC5 and MOPC21 (Litton Bionetics, Charleston, SC), was used in control experiments.

Gel electrophoresis and Western analysis. Synaptosomes were collected by FACS onto HA-type membrane filters $(0.45 \mu \mathrm{m}$ pore size; Millipore, Bedford, MA) under vacuum. Synaptosomes collected in this way accumulate on the filters in discrete spots that can be detected using an ultraviolet source. For SDS-PAGE, the spot containing the synaptosomes was cut out and transferred to an Eppendorf tube containing $50 \mu \mathrm{l}$ of Laemmli buffer. Samples were then sonicated, heated at $95^{\circ} \mathrm{C}$ for $10 \mathrm{~min}$, and centrifuged in a Beckman microfuge to pellet the paper. Preliminary experiments established that approximately $60 \%$ of the protein spotted onto Millipore filters is extracted under these conditions. Protein concentration was determined by the method of Peterson (1977). SDS-PAGE was performed on 0.8 -mm-thick $10 \%$ polyacrylamide slab gels by conventional methods (Laemmli, 1970). Proteins were electroblotted to nitrocellulose (Towbin et al., 1979). The Western blot was then incubated in $5 \%$ nonfat dry milk to block nonspecific antibody binding sites (Johnson et al., 1984) and reacted with $0.2 \mu \mathrm{g} / \mathrm{ml}$ of LNC $1 \mathrm{IgG}$. The antigen-antibody complex was visualized enzymatically with goat anti-mouse immunoglobulin conjugated to alkaline phosphatase (Blake et al., 1984). Relative amounts of discrete proteins in Western blots were quantified using a Zeineh soft laser scanning densitometer and accompanying software (Biomed Instruments, Fullerton, CA). SDSPAGE was also used to compare the electrophoretic mobilities of synaptosomal proteins fixed under various conditions (Fig. 6). Proteins were detected using Coomassie stain.

6-Hydroxydopamine lesions. Lesions were produced by bilateral administration of 6-hydroxydopamine (6-OHDA; Sigma) into the lateral ventricles using a modification of the method of Noble et al. (1967). Rats were anesthetized with chloral hydrate $(400 \mathrm{mg} / \mathrm{kg}$, i.p.). A hole was made $1.2 \mathrm{~mm}$ lateral to bregma on the coronal suture (Paxinos and Watson, 1982) using a $25 \mathrm{~s} / 8$-gauge needle with a stop $2-3 \mathrm{~mm}$ from its tip. The 6-OHDA was injected using a Hamilton syringe with a luer tip and a needle with a stop $4 \mathrm{~mm}$ from its tip. Each rat received $125 \mu \mathrm{g}$ of 6-OHDA per side, dissolved in $10 \mu \mathrm{l}$ of $0.9 \% \mathrm{NaCl}$ and $0.1 \%$ ascorbic acid. The rate of injection was approximately $2.5 \mu \mathrm{l} / \mathrm{min}$, and the syringe was allowed to remain in place for $1 \mathrm{~min}$ after the injection. Pargyline
$(50 \mathrm{mg} / \mathrm{kg}$, i.p.; Sigma) was administered $30 \mathrm{~min}$ before 6-OHDA treatment. This has been shown to enhance the toxicity of 6-OHDA (Breese and Traylor, 1970). Rats were killed $7 \mathrm{~d}$ after the lesion. DA levels were measured in aliquots of striatal homogenates to determine the extent of the lesion. Measurements were made using high-pressure liquid chromatography with electrochemical detection, essentially as described previously (Galloway et al., 1986).

\section{Results}

\section{Analysis of Zamboni-fixed synaptosomes}

Flow cytometric analysis and sorting based on intracellular antigens such as TH requires that synaptosomes be permeabilized by fixation to allow penetration by immunoglobulins and to immobilize intracellular antigens within permeabilized cells. Synaptosomes were fixed with modified Zamboni fluid (see Materials and Methods) and then compared with fresh synaptosomes with respect to forward angle light scatter (FALS) and $90^{\circ}$ light scatter (Fig. 1). FALS is proportional to particle size, while $90^{\circ}$ light scatter is a relative indicator of internal structure and granularity. As discussed in the accompanying paper (Wolf and Kapatos, 1989), light scatter analysis of fresh striatal synaptosomes indicated a heterogenous population of particles with diameters ranging from several tenths of a micron to several microns. Synaptosomes fixed with modified Zamboni fluid exhibited very similar light scattering properties, although the number of particles with high FALS signals was decreased slightly. This may suggest that larger synaptosomes are more susceptible to destruction during the repeated centrifugation and resuspension steps involved in the fixation process. Alternatively, synaptosomal structure may be modified by fixation so as to decrease the amount of light scattered at small angles. Zambonifixed synaptosomes (rinsed 4 times) were virtually identical to fresh synaptosomes with respect to autofluorescence (Fig. 1).

\section{Immunolabeling of dopaminergic synaptosomes}

Unlabeled fixed synaptosomes were used to define background fluorescence by setting an analysis window that contained only $0.2 \%$ of unlabeled synaptosomes. Synaptosomes that became sufficiently fluorescent to be included in this window as a result of immunolabeling were considered to have been recognized by the antibody in question. In order to label DA terminals selectively, fixed striatal synaptosomes were incubated sequentially with LNC 1 and a FITC-conjugated secondary antibody. FACS analysis demonstrated that this labeling procedure resulted in the appearance of a highly fluorescent subpopulation of synaptosomes. Dilution curves for LNC 1 ascites and control ascites fluid from the MOPC21 tumor line are shown in Figure 2. While nonspecific labeling by MOPC 21 increased with antibody concentration, specific labeling by LNC 1 (defined as the difference between labeling produced by LNC 1 and MOPC21) became maximal at a dilution of 1:2000, or approximately 2 $\mu \mathrm{g} / \mathrm{ml} \mathrm{IgG}$. This dilution was used in all subsequent experiments.

Dual parameter plots of FALS and fluorescence for unlabeled and LNC 1-labeled synaptosomes are presented in Figure 3. In this particular experiment, $13.1 \%$ of LNC 1-labeled synaptosomes were more fluorescent than unlabeled controls. If nonspecific labeling by a control monoclonal antibody $(2.7 \%)$ is subtracted, these results indicate that $10.4 \%$ of striatal synaptosomes were specifically labeled by LNC 1 . A similar percentage was found to be specifically labeled by LNC 1 when this experiment was repeated 4 times using separate preparations of striatal synaptosomes (mean $=9.9 \pm 0.5 \%$ ). This is consistent 


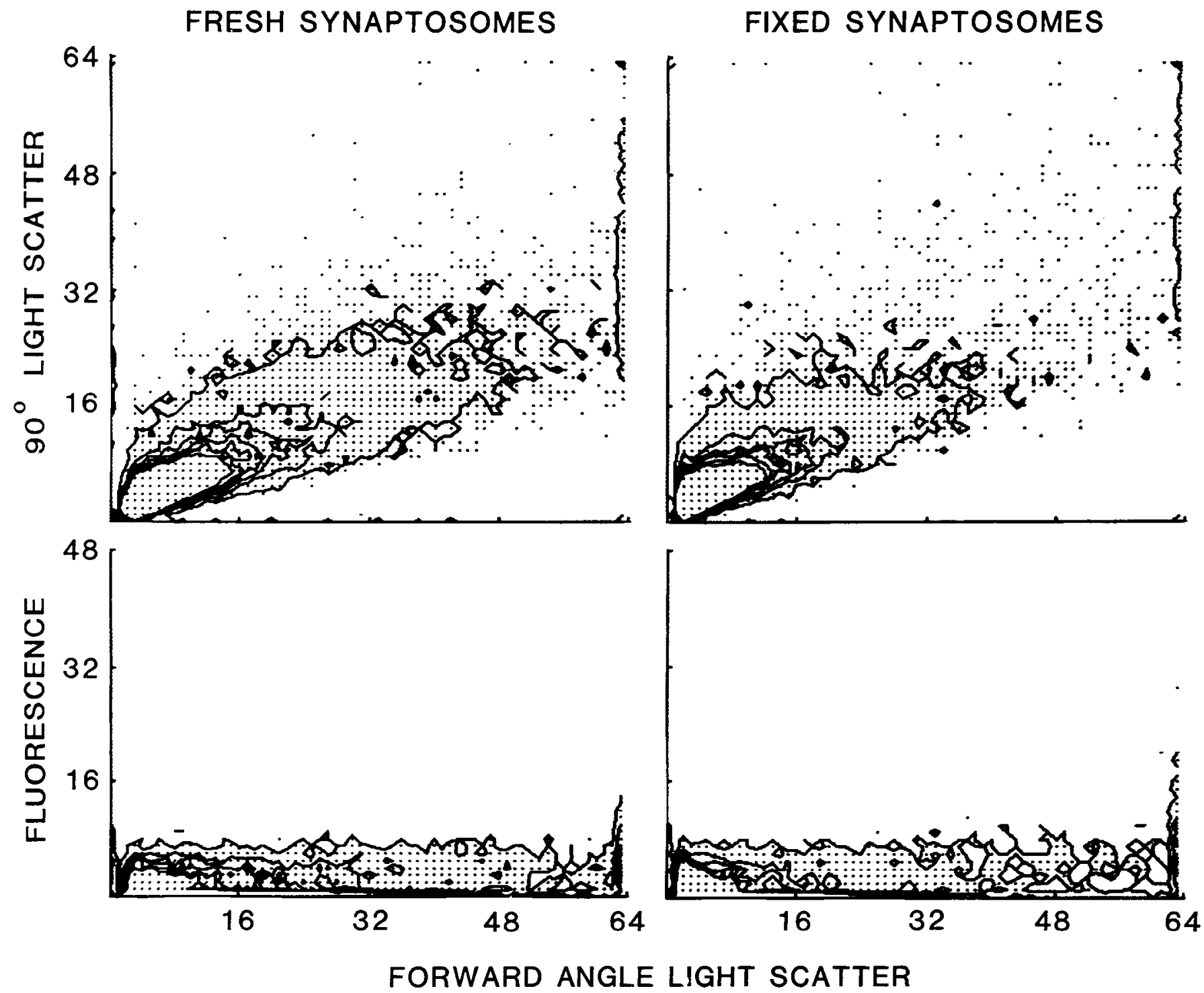

Figure 1. Comparison of light-scattering properties and fluorescence of fresh striatal synaptosomes and synaptosomes fixed with modified Zamboni fluid. Analysis was based on 20,000 events per sample.

with previous estimates, obtained using other methods, of the percentage of striatal synaptosomes that are dopaminergic (see Discussion). It is interesting that synaptosomes labeled by LNC 1 were not preferentially located in a particular range of FALS values (Fig. 3). This suggests that dopaminergic synaptosomes are heterogeneous with respect to size.

\section{Effect of 6-OHDA lesions on synaptosomal labeling}

If the synaptosomes labeled by LNC 1 are, in fact, dopaminergic, labeling should be decreased in synaptosomes prepared from rats pretreated with 6-OHDA so as to selectively destroy nigrostriatal DA terminals. We therefore compared the ability of LNC 1 and a control monoclonal antibody, RPC5, to label fixed striatal synaptosomes prepared from untreated and 6-OHDA lesioned rats. As predicted, 6-OHDA lesions resulted in significant decreases in LNC 1 labeling. This is illustrated in a dual parameter plot of FALS and fluorescence for synaptosomes prepared from a rat with an $82 \%$ depletion of striatal DA (Fig. 3). While $13.1 \%$ of striatal synaptosomes from an untreated rat were labeled by LNC 1, only $3.8 \%$ were labeled in synaptosomes prepared from the lesioned rat. Residual labeling by LNC 1 presumably occurred on surviving DA terminals.

FACS analysis of synaptosomes prepared from 4 rats with lesions of varying effectiveness indicated that the reduction in LNC 1 labeling was proportional to the extent of the lesion (Fig. 4). Regression analysis revealed a correlation coefficient of -0.93 between the percentage depletion of striatal DA and the percentage of striatal synaptosomes labeled by LNC 1. These findings suggest that synaptosomes labeled by LNC 1 are derived from the terminals of nigrostriatal DA neurons. Labeling by RPC5 was slightly increased after 6-OHDA lesions which pro-

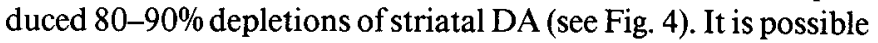
that additional nonspecific binding sites are exposed on the degenerating terminals or cellular debris that result from the lesion. Dual parameter analysis of FALS and $90^{\circ}$ light scatter revealed no significant differences between synaptosomes prepared from untreated and 6-OHDA-lesioned rats (data not shown), in support of our earlier conclusion that dopaminergic 
and nondopaminergic synaptosomes cannot be distinguished on the basis of light scattering properties.

\section{Analysis of TH content of sorted synaptosomes}

In order to verify that synaptosomes recognized by LNC 1 were enriched for $\mathrm{TH}$, equal numbers ( 3 million) of control and $\mathrm{LNC}$ 1-labeled synaptosomes were sorted onto membrane filters. Control synaptosomes (starting material) were sorted bascd on light scatter, while LNC 1-labeled synaptosomes (TH-positive synaptosomes) were sorted based on indirect immunofluorescence using a sort window set to include only $0.2 \%$ of control events. The proteins recovered from each filter were separated by SDS-PAGE, electroblotted to nitrocellulose, and immunoprobed for TH using LNC 1. In all lanes, LNC 1 recognized the $62 \mathrm{kDa}$ monomeric subunit of TH. Synaptosomes that had been sorted based on labeling with LNC 1 were found to contain approximately 6 times more TH than an equal number of control synaptosomes (Fig. 5).

\section{Comparison of fixation protocols}

As described above, Zamboni-fixed synaptosomes satisfied a number of criteria essential to these experiments: (1) They could be analyzed by FACS, suggesting that clumping was minimal; (2) they were very similar to fresh synaptosomes with respect to light scattering properties and autofluorescence; (3) fixation did not appear to interfere with recognition of TH by LNC 1 . An additional concern, however, is that fixation might produce significant modifications in other synaptosomal proteins, preventing their recognition by antibodies or other ligands and thereby interfering with attempts to further characterize DA terminals isolated by FACS. It was therefore of interest to determine whether synaptosomes could be permeabilized using gentler fixation conditions. To this end, synaptosomes fixed with modified Zamboni fluid were compared with synaptosomes fixed with low concentrations of paraformaldehyde alone with respect to (1) SDS-PAGE analysis of synaptosomal proteins and (2) immunolabeling of TH by LNC 1 as determined by FACS analysis.

SDS-PAGE analysis of proteins obtained from fresh synaptosomes, Zamboni-fixed synaptosomes, and synaptosomes fixed with varying concentrations of paraformaldehyde is shown in Figure 6 . The pattern of Coomassie staining is extremely similar in all lanes, suggesting that fixation does not alter the qualitative electrophoretic properties of synaptosomal proteins. However, even though equal amounts of protein were applied to all lanes, different amounts of protein appear to have been recovered from synaptosomes fixed by different methods. For example, frcsh synaptosomes contained several bands of high molecular weight that were less intense in synaptosomes fixed with high concentrations of paraformaldehyde or with modified Zamboni fluid. The lowest recovery of these high- $M_{\mathrm{r}}$ proteins was observed for Zamboni-fixed synaptosomes. Recovery improved with decreasing concentrations of paraformaldehyde alone, such that approximately equal amounts of all Coomassie-stained proteins were detected in fresh synaptosomes and synaptosomes fixed with low $(0.1$ and $0.01 \%)$ concentrations of paraformaldehyde. Examination of the amount of protein remaining in the stacking gel (see Fig. 6) suggested that lower recoveries were due to the fact that some protein never entered the resolving gel for samples fixed with modified Zamboni fluid or high concentrations of paraformaldehyde. This may be attributable to cross-linking of proteins as a result of fixation.

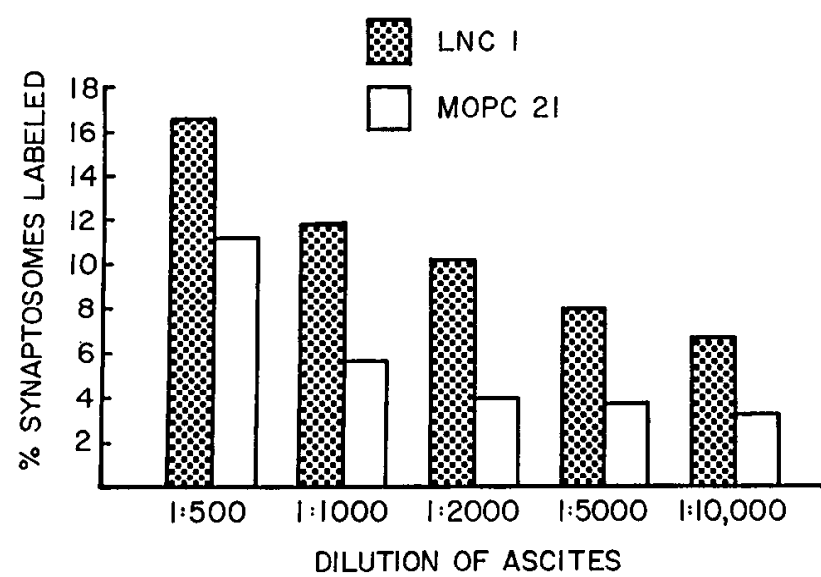

Figure 2. Dilution curves for LNC 1 and a control monoclonal antibody, MOPC21. Fixed striatal synaptosomes were incubated with the indicated dilution of ascites as described in Materials and Methods and then reacted with a 1:50 dilution of FITC-conjugated secondary antibody.

Next, FACS analysis was used to determine the extent to which TH was labeled by a 1:2000 dilution of LNC 1 in synaptosomes fixed with the methods described above. In order to define background fluorescence, an analysis window was set containing only $0.2 \%$ of fresh synaptosomes. Fixed synaptosomes falling into this window were considered to have been labeled by LNC 1. The greatest percentage of labeling was observed for Zamboni-fixed synaptosomes (11.6\%), with progressively fewer synaptosomes labeled at decreasing concentrations of paraformaldehyde (Table 1). However, labeling well above background levels $(5.1 \%)$ was observed even at the lowest concentration of paraformaldehyde $(0.01 \%)$. This suggests that all of the fixation protocols rendered synaptosomes permeable to antibodies. The decreased labeling observed with low concentrations of paraformaldehyde may suggest that cross-linking of membrane proteins was not sufficient to prevent soluble $\mathrm{TH}$ from diffusing out of the synaptosomes after fixation. This would be consistent with the observation that fixation methods which produced the greatest protein cross-linking, as assessed by failure of proteins to enter the resolving gel (Fig. 6), also resulted in the greatest percentage of synaptosomal labeling by LNC 1 .

\section{Discussion}

While synaptosomes have proven valuable in studies of synaptic function, their utility is limited by their chemical heterogeneity. This has prompted attempts to obtain synaptosomes enriched for various transmitters. Although a partial enrichment can be obtained in some cases based on differing sedimentation characteristics (see Robinson and Lovenberg, 1986), immunological approaches to defining synaptosomal subpopulations are most promising. For example, Richardson et al. (1984) obtained affinity-purified cholinergic terminals from rat brain using an antisera to a ganglioside antigen found on cholinergic membranes, enabling biochemical studies that would have been problematic in a mixed preparation (Richardson, 1986; Richardson and Brown, 1987; Richardson et al., 1987).

When utilized in combination with appropriate antibodies or other ligands, FACS has the potential to provide an elegant means of identifying and separating synaptosomal subpopulations (see Wolf and Kapatos, 1989). To date, neuroscientists have used FACS primarily to identify subpopulations of intact 


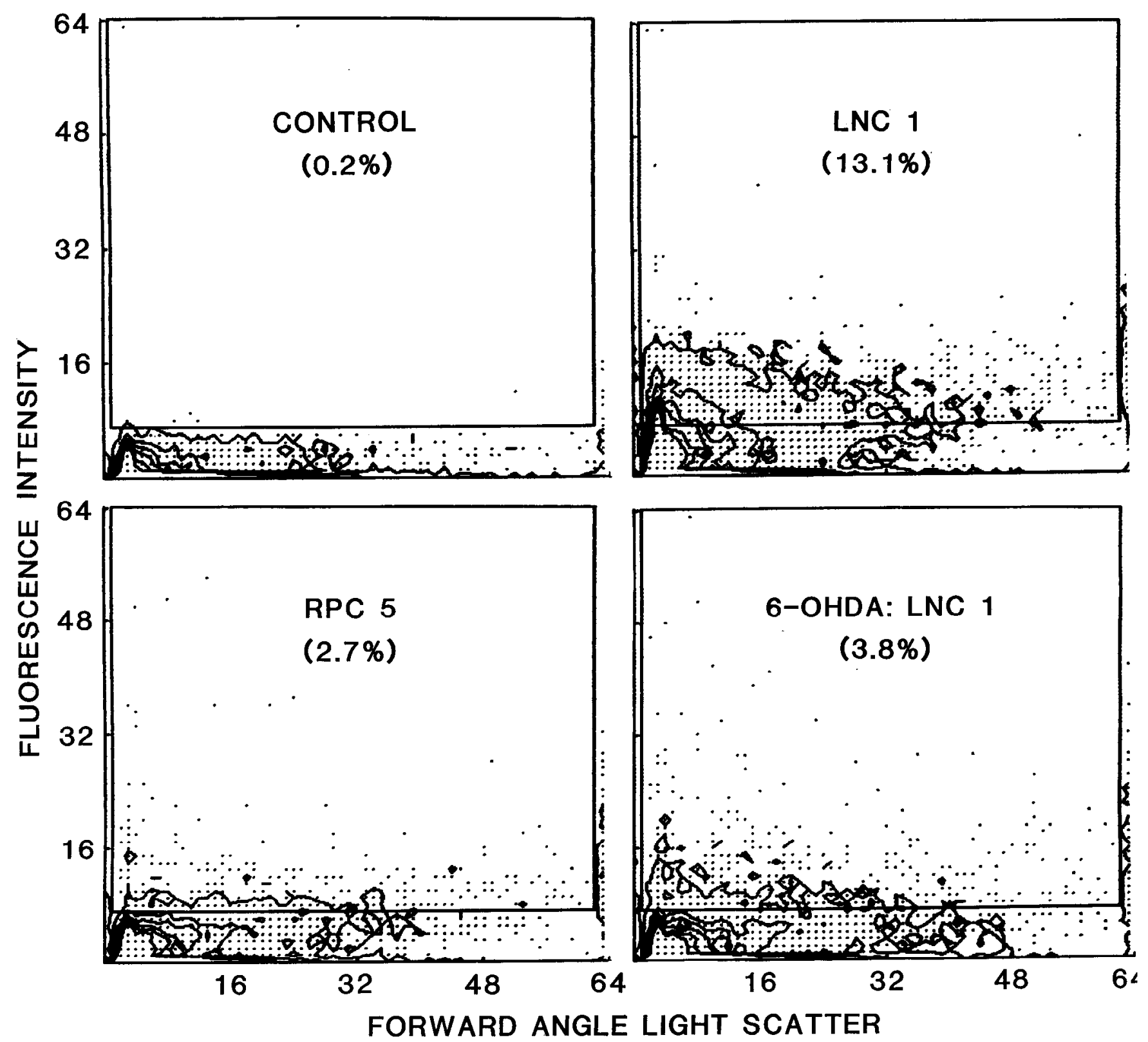

Figure 3. LNC 1 labeling of fixed striatal synaptosomes prepared from an untreated rat and a rat that received an intraventricular injection of 6-OHDA. The striatal DA content of the lesioned rat was $18 \%$ of control. The percentage of synaptosomes labeled in each group is indicated in parentheses. LNC 1 labeling is decreased considerably in lesioned tissue. Nonspecific binding was defined using a control monoclonal antibody, RPC5. A $4 \log$ unit range of fluorescence intensity is presented on the abscissa. Analysis was based on 20,000 events per sample.

cells based on differences in light scattering properties (Hatfield and Hymer, 1986a, b), the presence of cell surface antigens (Campbell et al., 1977; Rathjen et al., 1981; Abney et al., 1983; Barald, 1983; Derby et al., 1983; Dyer et al., 1983; Kapatos et al., 1983, 1985; Sack et al., 1983; Brackenbury et al., 1984; Moskal and Schaffner, 1986; St. John et al., 1986a, b; Chen et al., 1987; di Porzio et al., 1987), or the presence of retrogradely transported fluorescent markers (McPheeters and Okun, 1980; Armson and Bennett, 1983; Eagleson and Bennett, 1983; Rohrer et al., 1983; Calof and Reichardt, 1984; O'Brien and Fischbach, 1986; Schaffner et al., 1987).

Several recent reports have described the flow cytometric analysis of intracellular antigens in permeabilized cells. For ex- ample, indirect immunofluorescence techniques in combination with FACS have been used to determine the relative numbers of growth hormone-, prolactin-, and luteinizing hormone-producing cells in rat anterior pituitary (Hatfield and Hymer, 1985). In another study, fixed neurophysin-containing neurons, which comprised only $2 \%$ of the cells in the starting material, were isolated by FACS from dissociated tissue punches of rat supraoptic and paraventricular hypothalamic nuclei (Paden et al., 1986). Intracellular antigens have also been detected and quantified in lymphocytes and transformed cells (Zeile, 1980; Schroff et al., 1984; Freedman and Auersperg, 1986; Jacobberger et al., 1986; Young et al., 1986; Hayden et al., 1988). We now report the development of methods for the analysis and FACS-isola- 


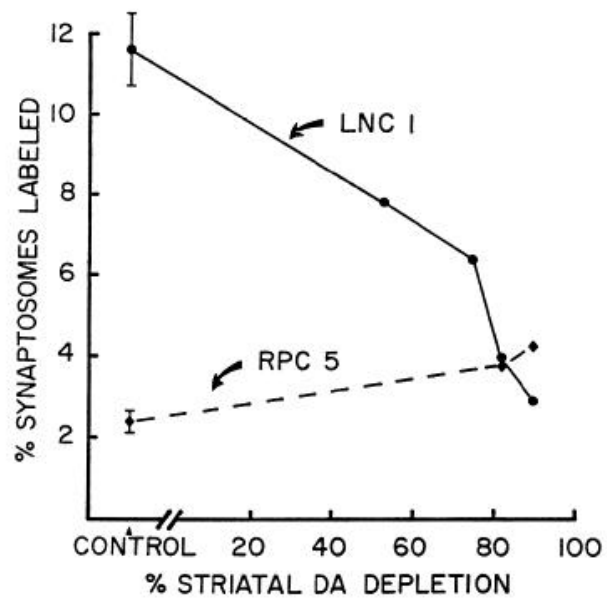

Figure 4. Analysis of LNC 1 labeling for fixed striatal synaptosomes prepared from control rats and from 4 rats with different levels of striatal DA depletion as a result of intraventricular injection of 6-OHDA.

tion of striatal synaptosomes that have been permeabilized by fixation and the use of these methods to obtain greatly enriched preparations of dopaminergic synaptosomes.

Dopaminergic striatal synaptosomes were identified based on the presence of $\mathrm{TH}$, an intracellular antigen confined to DA nerve terminals in this tissue (Swanson and Hartman, 1975). Analysis of 5 experiments indicated that $9.9 \pm 0.5 \%$ of the particles in fixed synaptosomal fractions of rat striatum were specifically labeled after sequential incubation with LNC 1 and FITC-secondary antibody. Data presented in the previous paper indicate that approximately $20-30 \%$ of the synaptosomal fraction represents particles other than intact synaptosomes, including free mitochondria and elements derived from astrocytes (Wolf and Kapatos, 1989). After correction for these contaminants, the present results suggest that $12-15 \%$ of fixed striatal
Table 1. Synaptosomal labeling by LNC 1: comparison of different methods of fixation

\begin{tabular}{lll} 
Treatment & Lanes (Fig. 6) & $\begin{array}{l}\text { \% Labeled } \\
\text { by LNC 1 }\end{array}$ \\
\hline Zamboni & $A$ & 11.6 \\
2\% paraformaldehyde & $B$ & 8.6 \\
1\% paraformaldehyde & $C$ & 8.2 \\
0.1\% paraformaldehyde & $D$ & 6.8 \\
0.01\% paraformaldehyde & $E$ & 5.1 \\
Fresh synaptosomes & $F$ & 3.2
\end{tabular}

Background fluorescence was defined as that level exceeded by only $0.2 \%$ of unlabeled fixed synaptosomes. Using this criteria, similar percentages of fresh synaptosomes were labeled by a 1:2000 dilution of LNC 1 (3.2\%), RPC5 (3.8\%), and MOPC21 $(3.9 \%)$, or after incubation with FITC-secondary antibody only (2.7\%).

synaptosomes contain TH. This is in close agreement with previous estimates of approximately $16 \%$ derived from fluorescence (Hökfelt, 1968; Hökfelt and Ungerstedt, 1969) and autoradiographic (Iversen and Schon, 1973) studies of catecholamine uptake into striatal nerve terminals. It should be noted that percentages of synaptosomes labeled by LNC 1 were derived by subtracting nonspecific labeling observed with a control monoclonal antibody of the same Ig class as LNC 1 and could, therefore, be underestimates if actual nonspecific labeling by LNC 1 is less than that produced by control monoclonal antibodies. Small variations in the percentage of striatal synaptosomes specifically labeled by LNC 1 in different experimental preparations probably reflect the difficulty of distinguishing between unlabeled terminals with high autofluorescence and dopaminergic terminals that for some reason (e.g., lower $\mathrm{TH}$ content) exhibit relatively low fluorescence intensity (see Fig. 3).

Control experiments demonstrated that LNC 1 labeling was

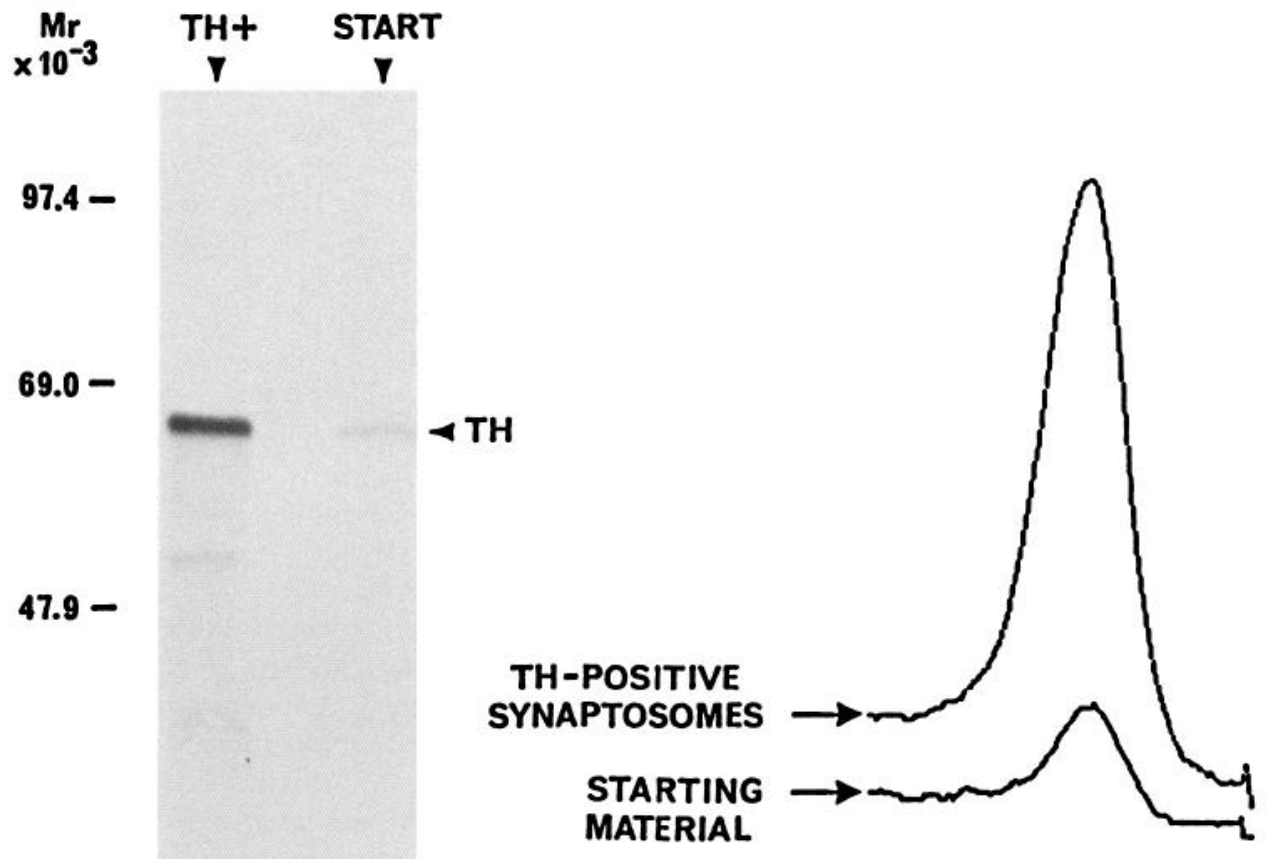

29.9
Figure 5. Comparison of TH levels in 3 million fixed control synaptosomes (starting material, or START) and 3 million fixed striatal synaptosomes sorted based on recognition by LNC 1 $(\mathrm{TH}+)$. Approximately $0.5 \mu \mathrm{g}$ of protein was applied to each lane, analyzed by SDS-PAGE, electroblotted, and immunoprobed with LNC 1. Densitometric analysis of the tyrosine hydroxylase band $(\mathrm{TH})$ is shown at right. 


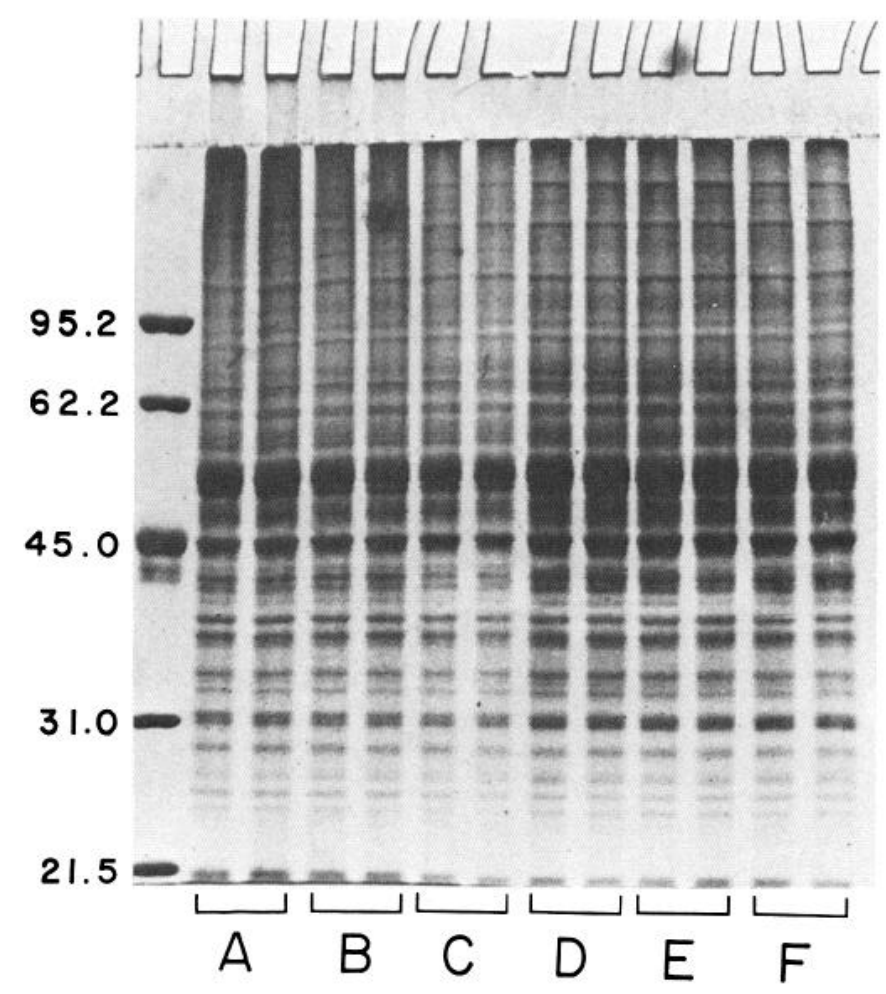

Figure 6. Comparison of proteins in fresh synaptosomes and synaptosomes fixed with different methods ( $A$, Zamboni; $B, 2 \%$ paraformaldehyde; $C, 1 \%$ paraformaldehyde; $D, 0.1 \%$ paraformaldehyde; $E, 0.01 \%$ paraformaldehyde; $F$, fresh synaptosomes). In each case, $20 \mu \mathrm{g}$ of synaptosomal protein was analyzed by SDS-PAGE in duplicate lanes and detected with Coomassie stain.

decreased in synaptosomes prepared from rats that had received intraventricular injections of 6-OHDA. The decrease in the percentage of synaptosomes labeled by LNC 1 was proportional to the percentage of striatal DA depletion produced by the lesion. These findings strongly suggest that the fluorescent subpopulation of synaptosomes observed after labeling with LNC 1 and FITC-secondary antibody was derived from nigrostriatal DA terminals. Synaptosomes sorted on the basis of recognition by LNC 1 were found to be enriched 6-fold for TH content compared with control synaptosomes sorted on the basis of light scatter. This is the degree of enrichment that would be predicted if TH-positive synaptosomes represented $16 \%$ of the starting material and if nearly all of the TH-positive synaptosomes were sorted using our protocol.

It is well known that fixation can have profound effects on antigenicity (Kerr et al., 1988). Thus, attempts to further characterize FACS-isolated dopaminergic synaptosomes could be hindered if fixation alters synaptosomal proteins of interest so as to interfere with their detection by antibodies or other ligands. Our results suggest that fixation with Zamboni fluid does not produce serious problems in this respect. While some degree of protein cross-linking occurred, this appears to be desirable and necessary when sorting based on soluble intracellular antigens like TH. Furthermore, Zamboni fixation does not alter the spectrum of synaptosomal proteins as detected by SDS-PAGE or their qualitative electrophoretic propertics. This indicates that Zamboni fixation will not prevent the identification of proteins on the basis of molecular weight.

Finally, it should be noted that it is not uncommon for an- tibodies raised against intact antigens to be used in the detection of these antigens in fixed tissue. With the exception of very small molecules, for which it is sometimes necessary to generate antibodies to the fixed form of the molecule, this is the approach that is generally used in histochemical studies. In fact, we have yet to encounter a case in which Zamboni fixation prevented an antibody from recognizing its appropriate antigen (unpublished observations). Similarly, results from other laboratories have shown that Zamboni fixation does not interfere with flow cytometric analysis of cells using antisera to growth hormone, prolactin, and luteinizing hormone (Hatfield and Hymer, 1985) or neurophysin (Paden et al., 1986).

It is interesting that the monomeric form of TH $(62 \mathrm{kDa})$ was identified in Western blots obtained from Zamboni-fixed synaptosomes. Previous studies have demonstrated that TH exists as a tetramer of identical subunits (Markey et al., 1980; Okuno and Fujisawa, 1982; Richtand et al., 1985). Since synaptosomes were fixed prior to sorting and SDS-PAGE, any oligomers that might have been cross-linked as a result of fixation would not have dissociated into monomers when subjected to denaturing gel electrophoresis. The detection of the monomeric form therefore suggests that fixation with modified Zamboni fluid does not produce extensive cross-linking of TH to other TH molecules (or to other proteins).

In the course of the present studies, we also attempted to develop flow cytometric methods for isolating intact dopaminergic synaptosomes. This could be accomplished readily if a cell surface marker unique to DA terminals could be identified and used to raise antibodies. Lacking such a marker, a number of alternatives were explored. Several neuronal populations have been successfully identified and sorted after being marked by retrograde transport of fluorescent tracers (see above for references). We attempted to introduce a fluorescent label into the terminals of nigrostriatal DA neurons using a FITC derivative of Phaseolus vulgaris leucoagglutinin (PHA-L), a plant lectin that is transported anterogradely. FITC-PHA-L was applied iontophoretically to the substantia nigra of adult rats using the method of Gerfen and Sawchenko (1984). After $12 \mathrm{~d}$, striatal synaptosomes were prepared from treated rats. When these synaptosomes were analyzed by FACS, a small number (1-2\%) of terminals were more fluorescent than controls (data not shown). This may suggest that FITC-PHA-L was taken up and transported by only a small fraction of the DA neurons in the substantia nigra. For example, if $10 \%$ of DA cell bodies took up the lectin and if DA terminals represent $15 \%$ of total terminals in the striatum, then only $1.5 \%$ of striatal terminals would be labeled. Unfortunately, subpopulations of this size are very difficult to analyze or sort. This approach is therefore problematic unless lectin could be introduced into the majority of the nigral DA neurons.

A second approach was based on the possibility that DA terminals might differ from other terminals in their cell surface carbohydrates. Subpopulations of striatal synaptosomes recognized by 11 FITC-conjugated plant lectins with different carbohydrate binding specificities were collected onto membrane filters by FACS and immunoprobed for TH using Western blot techniques. In several cases, TH was enriched or depleted in subpopulations of synaptosomes recognized by a particular lectin. However, no lectin labeled either dopaminergic or nondopaminergic synaptosomes exclusively (Wolf and Kapatos, 1989).

While the flow cytometric isolation of intact dopaminergic 
synaptosomes remains an exciting goal, there are some applications for which permeabilized synaptosomes are better suited. For example, it should be possible to detect and quantify intracellular antigens, including classical neurotransmitters, neuropeptides, and enzymes. Cell surface components, such as receptors and transporters, could also be examined. Since the FACS can detect fluorescent signals at 2 or more wavelengths, enabling simultaneous analysis of 2 or more antigens, this technique is well suited to colocalization studies. The question of whether a particular molecule is present in DA terminals could also be resolved by Western analysis of sorted TH-positive synaptosomes.

This approach should prove useful in evaluating theories about the role of specific molecules (e.g., components of signal transduction systems) in the regulation of dopaminergic function. Inherent in all such theories is the assumption that DA neurons actually contain the regulatory component in question. While histochemical methods can determine in a qualitative fashion whether a molecule is localized to a particular type of neuron, flow cytometry is unique in its ability to do so in a quantitative and statistically adequate manner. A stronger argument for a proposed chain of signal transduction could be made after showing that all the required molecular links are present in the appropriate neuronal population.

It is important to note that the techniques described in this paper can be applied to the analysis and isolation of any chemically identified population of nerve terminals or cells. Studies using human tissue are feasible given the recent demonstration that intact synaptosomes can be prepared from frozen human brain (Hardy et al., 1984; Haberland and Hetey, 1987a, b).

\section{References}

Abney, E. R., B. P. Williams, and M. C. Raff (1983) Tracing the development of oligodendrocytes from precursor cells using monoclonal antibodies, fluorescence-activated cell sorting, and cell culture. Dev. Biol. 100: 166-171.

Armson, P. F., and M. R. Bennett (1983) Neonatal retinal ganglion cell cultures of high purity: Effect of superior colliculus on their survival. Neurosci. Lett. 38: 181-186.

Barald, K. F. (1983) Monoclonal antibodies to chick ciliary ganglion isolate a neural crest subpopulation by fluorescence activated cell sorting. Soc. Neurosci. Abstr. 9: 342.

Blake, M. S., K. H. Johnston, G. J. Russell-Jones, and E. C. Gotschlich (1984) A rapid, sensitive method for detection of alkaline phosphatase-conjugated anti-antibody on western blots. Anal. Biochem. 136: 175-179.

Brackenbury, R., M. E. Greenberg, and G. M. Edelman (1984) Phenotypic changes and loss of N-CAM-mediated adhesion in transformed embryonic chicken retinal cells. J. Cell Biol. 99: 1944-1954.

Breese, G. R., and T. D. Traylor (1970) Effect of 6-hydroxydopamine on brain norepinephrine and dopamine: Evidence for selective degeneration of catecholamine neurons. I. Pharmacol. Exp. Ther. 174: $413-420$.

Calof, A. L., and L. F. Reichardt (1984) Motoneurons purified by cell sorting respond to two distinct activities in myotube-conditioned medium. Dev. Biol. 106: 194-210.

Campbell, G. LeM., M. Schachner, and S. O. Sharrow (1977) Isolation of glial cell-enriched and -depleted populations from mouse cerebellum by density gradient centrifugation and electronic cell sorting. Brain Res. 127: 69-86.

Chen, G.-G., P. A. St. John, and J. L. Barker (1987) Rat lactotrophs isolated by fluorescence-activated cell sorting are electrically excitable. Mol. Cell. Endocrinol. 51: 201-210.

Derby, M. A., S. A. Dyer, and L. Glaser (1983) Monoclonal antibodies against a differentiated retinal cell population. Brain Res. 283: 317325.

di Porzio, U., G. Rougon, E. A. Novotny, and J. L. Barker (1987)

Dopaminergic neurons from embryonic mouse mesencephalon are enriched in culture through immunoreaction with monoclonal antibody to neural specific protein 4 and flow cytometry. Proc. Natl. Acad. Sci. USA 84: 7334-7338.

Dyer, S. A., M. A. Derby, G. J. Cole, and L. Glaser (1983) Identification of sub-populations of chick neural retinal cells by monoclonal antibodies: A fluorescence activated cell sorter screening technique. Brain Res. 285: 197-203.

Eagleson, K. L., and M. R. Bennett (1983) Survival of purified motor neurones in vitro: Effects of skeletal muscle-conditioned medium. Neurosci. Lett. 38: 187-192.

Freedman, D., and N. Auersperg (1986) Detection of an intracellular transforming protein ( $\mathrm{v}-\mathrm{Ki}$-ras $\mathrm{p} 21)$ using the flow activated cell sorter (FACS). In Vitro Cell. Dev. Biol. 22: 621-624.

Galloway, M. P., M. E. Wolf, and R. H. Roth (1986) Regulation of dopamine synthesis in the medial prefrontal cortex is mediated by release modulating autoreceptors: Studies in vivo. J. Pharmacol. Exp. Ther. 236: 689-698.

Gerfen, C. R., and P. E. Sawchenko (1984) An anterograde neuroanatomical tracing method that shows the detailed morphology of neurons, their axons and terminals: Immunohistochemical localization of an axonally transported plant lectin, Phaseolus vulgaris Leucoagglutinin (PHA-L). Brain Res. 290: 219-238.

Gray, E. G., and V. P. Whittaker (1962) The isolation of nerve endings from brain: An electron-microscopic study of cell fragments derived by homogenization and centrifugation. J. Anat. 96: 79-88.

Haberland, N., and L. Hetey (1987a) Studies in postmortem dopamine uptake. I. Kinetic characterization of the synaptosomal dopamine uptake in rat and human brain after postmortem storage and cryopreservation. Comparison with noradrenaline and serotonin uptake. J. Neural Transm. 68: 289-301.

Haberland, N., and L. Hetey (1987b) Studies in postmortem dopamine uptake. II. Alterations of the synaptosomal catecholamine uptake in postmortem brain regions in schizophrenia. J. Neural Transm. 68: 303-313.

Hardy, J. A., P. R. Dodd, A. E. Oakley, R. H. Perry, J. A. Edwardson, and A. M. Kidd (1983) Metabolically active synaptosomes can be prepared from frozen rat and human brain. J. Neurochem. 40:608614.

Hatfield, J. M., and W. C. Hymer (1985) Flow cytometric immunofluorescence of rat anterior pituitary cells. Cytometry 6: 137-142.

Hatfield, J. M., and W. C. Hymer (1986a) Flow cytometric analysis and sorting of live male rat anterior pituitary cell types by forward angle and perpendicular light scatter. Endocrinology 119:2670-2682.

Hatfield, J. M., and W. C. Hymer (1986b) Flow cytometric analysis and sorting of live female rat anterior pituitary cell types by forward angle and perpendicular light scatter: Effect of $17 \beta$-estradiol. Endocrinology 119: 2683-2694.

Hayden, G. E., K. Z. Walker, J. F. A. P. Miller, J. S. Wotherspoon, and R. L. Raison (1988) Simultaneous cytometric analysis for the expression of cytoplasmic and surface antigens in activated $T$ cells. Cytometry 9: 44-51.

Hökfelt, T. (1968) In vitro studies on central and peripheral monoamine neurons at the ultrastructural level. Z. Zellerforsch. 91:1-74.

Hökfelt, T., and U. Ungerstedt (1969) Electron and fluorescence microscopical studies on the nucleus caudatus putamen of the rat after unilateral lesions of ascending nigro-neostriatal dopamine neurons. Acta Physiol. Scand. 76: 415-426.

Iversen, L. L., and F. E. Schon (1973) The use of autoradiographic techniques for the identification and mapping of transmitter-specific neurones in CNS. In New Concepts in Neurotransmitter Regulation. A. J. Mandell, ed., pp. 153-193, Plenum, New York.

Jacobberger, J. W., D. Fogleman, and J. M. Lehman (1986) Analysis of intracellular antigens by flow cytometry. Cytometry $7: 356-364$.

Johnson, D. A., J. W. Gautsch, J. R. Sportsman, and J. H. Elder (1984) Improved technique utilizing nonfat dried milk for analysis of proteins and nucleic acids transferred to nitrocellulose. Gene Anal. Techn. 1: 3-8.

Kapatos, G., M. T. Caserta, and J. L. Barker (1983) A monoclonal antibody (mAb) produced by in vitro immunization against clonal pituitary cells recognizes a surface protein on specific populations of neurons in the mouse CNS. Soc. Neurosci. Abstr. 9: 343.

Kapatos, G., J. Massetta, and J. L. Barker (1985) A monoclonal antibody that recognizes a cell-surface protein on $\mathrm{GH}_{3}$ cells. In Prolactin. Basic and Clinical Correlates, R. M. MacLeod, M. O. Thorner, and U. Scapagnini, eds., pp. 45-52. Liviana Press, Padua.

Kerr, R. S. C., A. H. Kaye, and P. F. Bartlett (1988) A quantitative 
assessment by flow cytometry of the preservation of the Thy-1 antigen by various fixation procedures. Neurosci. Lett. 87: 250-265.

Kruth, H. S. (1982) Flow cytometry: Rapid biochemical analysis of single cells. Anal. Biochem. 125: 225-242.

Laemmli, U. K. (1970) Cleavage of structural proteins during the assembly of the head of bacteriophage T4. Nature 227: 680-685.

Loken, M. R., and A. M. Stall (1982) Flow cytometry as an analytical and preparative tool in immunology. J. Immunol. Methods 50: R85R112.

Markey, K. A., S. Kondo, L. Shenkman, and M. Goldstein (1980) Purification and characterization of tyrosine hydroxylase from a clonal pheochromocytoma cell line. Mol. Pharmacol. 17: 79-85.

McPheeters, M., and L. M. Okun (1980) Identification and isolation in vitro of presumptive motoneurons marked by retrograde transport of a new fluorescent tracer. Soc. Neurosci. Abstr. 6: 733.

Moskal, J. R., and A. E. Schaffner (1986) Monoclonal antibodies to the dentate gyrus: Immunocytochemical characterization and flow cytometric analysis of hippocampal neurons bearing a unique cellsurface antigen. J. Neurosci. 6: 2045-2053.

Noble, E. P., R. J. Wurtman, and J. Axelrod (1967) A simple and rapid method for injecting $\mathrm{H}^{3}$-norepinephrine into the lateral ventricle of the rat brain. Life Sci. 6: 281-291.

O'Brien, R. J., and G. D. Fischbach (1986) Isolation of embryonic chick motoneurons and their survival in vitro. J. Neurosci. 6: $3265-$ 3274 .

Okuno, S., and H. Fujisawa (1982) Purification and some properties of tyrosine 3-monooxygenase from rat adrenal. Eur. J. Biochem. 122: $49-55$.

Paden, C. M., D. L. Berglund, S. J. Hapner, and C. J. Welsh (1986) A flow cytometric method for intracellular labeling and purification of rare neuronal populations: Isolation of fixed neurophysin neurons. Brain Res. 376: 310-319.

Paxinos, G., and C. Watson (1982) The Rat Brain in Stereotaxic Coordinates, Academic, New York.

Peterson, G. L. (1977) A simplification of the protein assay method of Lowry et al. which is more generally applicable. Anal. Biochem. 83: 346-356.

Rathjen, F. G., B. Holcombe, and A. Gierer (1981) Separation of cell populations from embryonic chick neural retina with fluorescenceactivated cell sorting. J. Biochem. Biophys. Methods 4: 191-207.

Richardson, P. J. (1986) Choline uptake and metabolism in affinitypurified cholinergic nerve terminals from rat brain. J. Neurochem. 46: $1251-1255$.

Richardson, P. J., and S. J. Brown (1987) ATP release from affinitypurified rat cholinergic nerve terminals. J. Neurochem. 48: 622-630.

Richardson, P. J., K. Siddle, and J. P. Luzio (1984) Immunoaffinity purification of intact, metabolically active, cholinergic nerve terminals from mammalian brain. Biochem. J. 219:647-654.
Richardson, P. J., S. J. Brown, E. M. Bailyes, and J. P. Luzio (1987) Ectoenzymes control adenosine modulation of immunoisolated cholinergic synapses. Nature $327: 232-234$.

Richtand, N. M., T. Inagami, K. Misono, and R. Kuczenski (1985) Purification and characterization of rat striatal tyrosine hydroxylase. Comparison of the activation by cyclic AMP-dependent phosphorylation and by other effectors. J. Biol. Chem. 260:8465-8473.

Robinson, P. J., and W. Lovenberg (1986) Dopamine and serotonin in two populations of synaptosomes isolated by Percoll gradient centrifugation. Neurochem. Int. 9: 455-458.

Rohrer, D. C. D., D. M. Gash, M. F. D. Notter, and J. F. Leary (1983) Isolation of fluorescence-labelled hypothalamic neurons. Soc. Neurosci. Abstr. 9: 303.

Sack, H. J., M. Stohr, and M. Schachner (1983) Cell-type specific binding of Ricinus lectin to murine cerebellar cell surfaces in vitro. Cell Tissue Res. 228: 183-204.

Schaffner, A. E., P. A. St. John, and J. L. Barker (1987) Fluorescenceactivated cell sorting of embryonic mouse and rat motoneurons and their long-term survival in vitro. J. Neurosci. 7: 3088-3104.

Schroff, R. W., C. D. Bucana, R. A. Klein, M. M. Farrell, and A. C. Morgan, Jr. (1984) Detection of intracytoplasmic antigens by flow cytometry. J. Immunol. Methods 70: 167-177.

Stefanini, M., C. De Martino, and L. Zamboni (1967) Fixation of ejaculated spermatozoa for electron microscopy. Nature 216: 173174.

St. John, P. A., W. M. Kell, J. S. Mazzetta, G. D. Lange, and J. L. Barker (1986a) Analysis and isolation of embryonic mammalian neurons by fluorescence-activated cell sorting. J. Neurosci. 6: 14921512.

St. John, P. A., L. Dufy-Barbe, and J. L. Barker (1986b) Anti-prolactin cell-surface immunoreactivity identifies a subpopulation of lactotrophs from the rat anterior pituitary. Endocrinology 119:2783-2795.

Swanson, L. W., and B. K. Hartman (1975) The central adrenergic system. An immunofluorescence study of the location of cell bodies and their efferent connections in the rat utilizing dopamine- $\beta$-hydroxylase as a marker. J. Comp. Neurol. 163: 467-505.

Towbin, H., T. Staehlein, and J. Gordon (1979) Electrophoretic transfer of proteins from polyacrylamide gels to nitrocellulose sheets: Procedure and some applications. Proc. Natl. Acad. Sci. USA 76: 43504354.

Wolf, M. E., and G. Kapatos (1989) Flow cytometric analysis of rat striatal nerve terminals. J. Neurosci. 9: 94-105.

Young, H. A., R. A. Klein, T. Y. Shih, A. C. Morgan, Jr., and R. W. Schroff (1986) Detection of the intracellular ras p21 oncogene product by flow cytometry. Anal. Biochem. 156: 67-71.

Zeile, G. (1980) Intracytoplasmic immunofluorescence in multiple myeloma. Cytometry $1: 37-41$. 